syndrome. A multicenter report. Journal of the American College of Cardiology, 20, 1391-1396.

Buckley, N. A. \& Sanders, P. (2000) Cardiovascular adverse effects of antipsychotic drugs. Drug Safety, $\mathbf{2 3}$ 215-228

Drici, M. D., Wang, W. X., Liu X. E., et al (1998) Prolongation of QT interval in isolated feline hearts by antipsychotic drugs. Journal of Clinical Psychopharmacology, 18, 477-481.

Jefferson, J.W. (1998) Drug and diet interactions avoiding therapeutics paralysis. Journal of Clinical Psychiatry, 59 (suppl. 16), 31-39.

S.-A.Chong Institute of Mental Health and Woodbridge Hospital, 10 Buangkok View, Singapore 539747, Singapore

\section{Cognitive-behavioural techniques in practice}

The randomised controlled trial reported by Turkington $\&$ Kingdon (2000) demonstrated therapeutic benefit from a general psychiatrist using cognitive-behavioural techniques with patients experiencing psychotic symptoms. However, the conclusion that "general psychiatrists could help their patients with schizophrenia by using cognitive-behavioural techniques" (p. 101) may not fully reflect the fact that the sole therapist in this study is an international expert in cognitive-behavioural therapy (CBT) with psychosis, who has co-written one of the seminal texts (Kingdon \& Turkington, 1994).

Lack of time is highlighted as the main difficulty for psychiatrists wanting to use CBT, but other limiting factors may include the knowledge, skills and attitudes of the psychiatrist. Experience as a clinical psychologist supervising trainee psychiatrists suggests that the knowledge base is not generally problematic-medical training equips practitioners with the ability to assimilate new information rapidly.

Skills development in CBT is more difficult. The difference between the Turkington \& Kingdon approach and cognitive therapy is that a formulation is not used, but it may be that an experienced CBT practitioner (as in their study) employs an implicit individualised formulation, whereas a more typical general psychiatris would not. Cognitive-behavioural techniques can be powerful, and using these techniques without a formulation can be clinically dangerous. For example, undertaking attributional change in a patient with paranoia without regard for the link between persecutory delusions and selfesteem (Lyon et al, 1994) may trigger depression. Cognitive-behavioural skills development requires clinical supervision.

Attitudinal change is most difficult. Consider the response of a psychiatrist to a patient's question, "Should I take my medication?". Valid responses might include "Yes" from a biological psychiatrist, and "Here's the evidence of effectiveness ..." from a social psychiatrist. However, a response such as "How will you decide?" is a more appropriate cognitive-behavioural response. Psychiatrists can develop these alternative attitudes, but a 'psychiatric' mind-set often re-emerges in therapy.

Improved access for patients to psychological therapies is imperative. However, although it may serve the profession of psychiatry well to indicate that the use of CBT is easily within the grasp of all its practitioners, it is not clear that this is empirically demonstrated.

Kingdon, D. \& Turkington, D. (1994) CognitiveBehavioral Therapy of Schizophrenia. Hove: Lawrence Erlbaum

Lyon, H. M., Kaney, S. \& Bentall, R. P. (1994) The defensive function of persecutory delusions. Evidence from attribution tasks. British Journal of Psychiatry, 164 637-646

Turkington, D. \& Kingdon, D. (2000) Cognitivebehavioural techniques for general psychiatrists in the management of patients with psychoses. British Journal of Psychiatry, 177, I0I-106.

M. Slade Health Services Research Department, Institute of Psychiatry, Denmark Hill, London SE5 8AF

Authors' reply: We thank Dr Slade for his interest in our paper and are grateful for the chance to clarify these crucial points. At the time this study was carried out D.K. was a general psychiatrist working full-time in the community. He did have a general cognitive therapy training and applied the techniques that he had learned for the treatment of depression and anxiety in an adapted form to his patients with schizophrenia (Kingdon \& Turkington, 1991). We then designed this study to test the efficacy of these techniques as against a befriending control (Kingdon et al, 1989). The study was then carried out in the course of D.K.'s clinical work.

We accept that a shared formulation is a fundamental component of cognitive therapy with schizophrenia (Fowler et al, 1995), which not only helps to direct the process of therapy but which can also help to predict the emergence of depression as a delusion recedes. The identification of key maladaptive core beliefs is part of the process of formulation and helps when there are blocks in progress and in the prevention of relapse. Psychiatrists do not normally identify such core beliefs in their case formulations and may not share all of the formulation with the patient. However, the formulations of psychiatrists do contain an aetiological component comprising such issues as genetic predisposition, birth traumas, early losses and personality. The precipitation of the psychosis in relation to any pertinent life events is included, as are maintaining factors such as isolation, poor adherence or high expressed emotion within the family (Gelder et al, 1983). Formulation is so central to psychiatric practice that it is a key component of the Royal College of Psychiatrists' membership examination. With such formulations psychiatrists can safely use cognitive-behavioural techniques as long as they remain aware of the risk of emerging depression or increased suicidal ideation linked to improved insight. Such phenomena are widely recognised in psychiatry when certain types of symptoms (e.g. grandiose or systematised delusions) respond to antipsychotic medication.

The purpose of our paper was to attempt to shift attitudes in psychiatry in order that we can be better engaged with our patients who have psychoses by working more directly with their symptoms rather than simply monitoring them and titrating antipsychotic medication. If general psychiatrists can make this shift in attitude, training workshops and supervision will be necessary. We expect that this shift could well facilitate the delivery of formal cognitive therapy by other community mental health teams trained in this approach and help with the implementation of other psychosocial interventions in this patient population.

Fowler, D., Garety, P. \& Kuipers, E. (1995) Cognitive Behaviour Therapy for Psychosis: Theory and Practice. The Wiley Series in Clinical Psychology. Chichester: John Wiley \& Sons.

Gelder, M., Gath, D. \& Mayou, R. (1983) Oxford Textbook of Psychiatry. Oxford: Oxford Medical.

Kingdon, D. G. \& Turkington, D. (1991) Preliminary report: the use of cognitive behaviour therapy and a normalizing rationale in schizophrenia. Journal of Nervous and Mental Disease, I79, 207-2II.

_, _ Collis, J., et al (1989) Befriending: cost effective community care. Psychiatric Bulletin, I3, 350-351.

D. Turkington, D. G. Kingdon Emergency Liaison Team, Leazes Wing, Royal Victoria Infirmary, Queen Victoria Road, Newcastle upon Tyne NEI 4LP 